\title{
Magneto-optical study of the spin-polarized electronic states in multiferroic $\mathrm{TbMnO}_{3}$
}

\author{
M. Bastjan, ${ }^{1}$ S. G. Singer, ${ }^{1}$ G. Neuber, ${ }^{1}$ S. Eller, ${ }^{1}$ N. Aliouane, ${ }^{2}$ D. N. Argyriou, ${ }^{2}$ S. L. Cooper, ${ }^{3}$ and M. Rübhausen ${ }^{1}$ \\ ${ }_{1}^{1}$ Institut für Angewandte Physik, Universität Hamburg, Jungiusstraße 11, D-20355 Hamburg, Germany \\ ${ }^{2}$ Hahn-Meitner Institut, Glienicker Straße 100, D-14109 Berlin, Germany \\ ${ }^{3}$ Frederick Seitz, Materials Research Laboratory and Department of Physics, University of Illinois at Urbana-Champaign, Urbana, \\ Illinois 61801, USA
}

(Received 7 March 2008; revised manuscript received 9 April 2008; published 7 May 2008)

\begin{abstract}
The magnetic and electronic properties of multiferroic $\mathrm{TbMnO}_{3}$ in the paramagnetic, antiferromagnetic, sinusoidal, and spiral-spin phases were studied by spectral generalized magneto-optical ellipsometry. The measurements show a strong anisotropy of the dielectric tensor. A redistribution of spectral weight was observed in the diagonal components of the dielectric tensor for the temperature range from 110 to $T_{N}=46 \mathrm{~K}$. In the off-diagonal elements, spectral generalized magneto-optical ellipsometry shows sensitivity to the antiferromagnetic and ferroelectric phase transitions at $T_{N}=46 \mathrm{~K}$ and $T_{F}=29 \mathrm{~K}$, respectively, and a persistent signal up to $6 T_{N}$.
\end{abstract}

DOI: 10.1103/PhysRevB.77.193105

PACS number(s): 75.47.Lx, 75.25.+z, 75.50.Ee, 77.22.Ej

Multiferroic materials exhibit phases with simultaneous magnetic and ferroelectric properties. The close coupling of the magnetization and the electric polarization in multiferroics makes these materials excellent candidates for demonstrating giant magnetoelectrical behavior, ${ }^{1,2}$ in which large magnetic and electric responses can be induced with weak electric and magnetic fields, respectively. ${ }^{3,4}$ Spectral generalized magneto-optical ellipsometry (SGME) is an ideal technique for studying the interplay between electric and magnetic properties in multiferroic systems, as it probes both the diagonal $\varepsilon_{x x}$ and off-diagonal $\varepsilon_{x y}$ components of the dielectric tensor. ${ }^{5}$

Multiferroicity in $\mathrm{TbMnO}_{3}$ is induced by spin-charge coupling. Below the Néel temperature $T_{N}=46 \mathrm{~K}$, the wavelength of the incommensurate spin density decreases with decreasing temperature down to the ferroelectric transition at $T_{F}$ $=29 \mathrm{~K}$. In the absence of a magnetic field, this spin-density modulation gets locked in at $T_{F}$. Below $T_{F}$, the inversion symmetry of the lattice is broken by the $b c$-cycloidal arrangement of the spins, inducing a ferroelectric polarization along the $c$ axis while the antiferromagnetic ordering along the $b$ axis persists. $3,4,6$

Here, we study the coupling between spin and charge degrees of freedom in multiferroic $\mathrm{TbMnO}_{3}$ by examining the temperature-dependent dielectric responses $\varepsilon_{x x}$ and $\varepsilon_{x y}$, which are the diagonal and off-diagonal components of the dielectric tensor. The magnetization of a material breaks the symmetry between left and right circularly polarized (LCP and RCP) light. While $\varepsilon_{2 x x}$ is proportional to the sum of absorptions of LCP and RCP light, $\varepsilon_{1 x y}$ is proportional to the difference. ${ }^{7}$ Consequently, a complete magneto-optical characterization of $\varepsilon_{2 x x}$ and $\varepsilon_{1 x y}$ in a magnetic material provides detailed information regarding the coupling between the material's electronic and magnetic properties.

The temperature-dependent ellipsometry was done with a custom-made setup by using an extended Sentech SE850 spectral ellipsometer inside a He flow cryostat. The cryostat has stress-free mounted windows and maintains a base pressure of about $3 \times 10^{-8}$ mbar at room temperature. ${ }^{8}$ Standard ellipsometry was performed for temperatures from 10 to $470 \mathrm{~K}$ in the spectral range from 0.5 to $5.5 \mathrm{eV}$. In
SGME, one measures the fractional difference of the intensities of light at a particular polarization angle for magnetic fields, which is applied along opposite directions, i.e., $\delta I / I$ $=2\left(I_{-}-I_{\uparrow}\right) /\left(I_{+}+I_{\uparrow}\right) .8,9$ A magnetic field $H= \pm 440 \mathrm{mT}$ was applied parallel to the normal vector of the plane of incidence. SGME measurements were performed in the spectral range from 1.6 to $5.5 \mathrm{eV}$ for temperatures from 15 to $300 \mathrm{~K}$. For measurements on the $\mathrm{BC}$ plane, two identical lenses were mounted in order to reduce the spot size to 100 $\times 300 \mu \mathrm{m}^{2}$.

The $\mathrm{TbMnO}_{3}$ crystals were grown at the Hahn-Meitner Institut in a floating zone image furnace under $\mathrm{Ar}$ atmosphere. The phase purity of these crystals was checked by performing x-ray and neutron diffraction measurements on a small part of each sample. The resulting diffraction patterns indicate that the samples were single phase and orthorhombic. The specific heat and superconducting quantum interference device (SQUID) measurements show three successive transitions at $T_{N}(\mathrm{Mn})=46 \mathrm{~K}, T_{F}=29 \mathrm{~K}$, and $T_{N}(\mathrm{~Tb})=7 \mathrm{~K}$, which are in good agreement with published data. ${ }^{2}$ The $\mathrm{TbMnO}_{3}$ samples have dimensions of $6 \times 5 \times 2$ and $5 \times 3$ $\times 2 \mathrm{~mm}^{3}$. They were polished on an exposed $\mathrm{AB}$ and $\mathrm{BC}$ planes.

In Figs. 1(a) and 1(c), we present the real $\left(\varepsilon_{1 x x}\right)$ and imaginary $\left(\varepsilon_{2 x x}\right)$ parts of the diagonal elements of the dielectric tensor of $\mathrm{TbMnO}_{3}$. The quantity $\varepsilon^{c}$ denotes the dielectric function in the $c$-axis direction. For both planes, the temperature range between 10 and $450 \mathrm{~K}$ was investigated. The spectra for the $\mathrm{BC}$ plane were taken along the $c$ axis [Fig. $1(\mathrm{c})]$ and a direction $17^{\circ}$ off the $b$ axis [Fig. 1(a)]. Figure 1(b) shows $\varepsilon_{1 x x}$ and $\varepsilon_{2 x x}$ on the $\mathrm{AB}$ plane along a direction $25^{\circ}$ off the $b$ axis, for the same temperatures. While the measurements close to the $b$ axis, on both planes, show bands centered around 2 and $5 \mathrm{eV}$ [Figs. 1(a) and 1(b)], only the $5 \mathrm{eV}$ band is present for $\varepsilon_{\mathrm{xx}}^{c}$ [Fig. 1(c)]. The band at $2 \mathrm{eV}$ exhibits an anisotropy and a temperature dependence. The origin of the $2 \mathrm{eV}$ band in undoped manganites has been studied by several authors. ${ }^{10-15}$ In more detail, the $2 \mathrm{eV}$ band consists of three separate peaks at roughly 1.9, 2.3, and $2.6 \mathrm{eV}$. At low temperatures, for measurements on the $\mathrm{AB}$ 

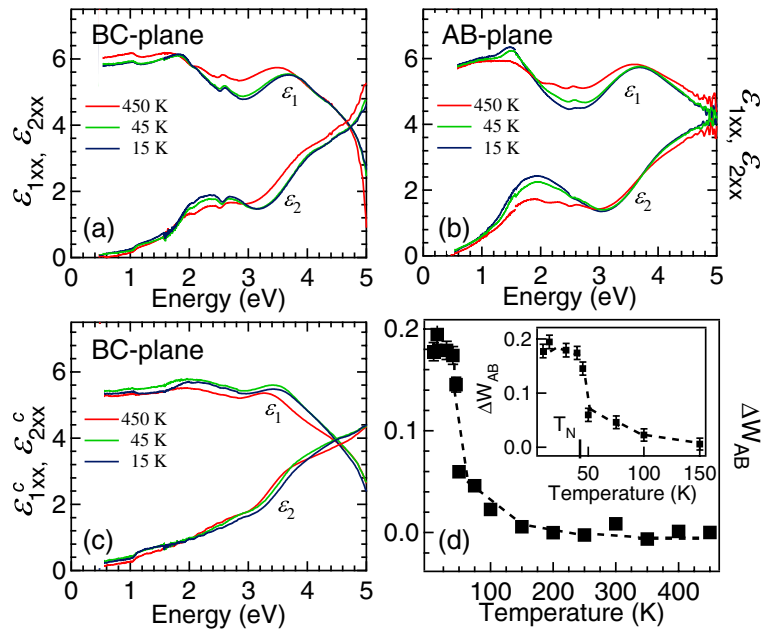

FIG. 1. (Color online) Diagonal elements $\left(\varepsilon_{x x}\right)$ of the dielectric tensor of $\mathrm{TbMnO}_{3}$ for 450 (red), 45 (green), and $15 \mathrm{~K}$ (blue), which are measured on the $\mathrm{BC}[(\mathrm{a})$ and $(\mathrm{c})]$ as well as on the $\mathrm{AB}$ plane [see (b)]. The $\varepsilon_{1 x x}$ and $\varepsilon_{2 x x}$ are the real and the imaginary parts of the dielectric function. (d) shows the changes in the normalized integrated spectral weight as a function of temperature for the $A B$ plane. The inset displays details of these changes in temperatures between 10 and $150 \mathrm{~K}$.

plane close to the $b$ axis, the appearance of three separate peaks is less pronounced [see Fig. 1(b)]. In recent publications, Kovaleva et al. ${ }^{13}$ and Kim et al. ${ }^{14,15}$ assigned this $2 \mathrm{eV}$ band to intersite $\mathrm{Mn}^{3+} d-d$ transitions. In contrast, the band at $5 \mathrm{eV}$ is mainly isotropic and can be associated with the manifold of charge transfer transitions from $\mathrm{O} 2 p \rightarrow \mathrm{Mn} 3 d$ levels starting around $3 \mathrm{eV}$ and extending to much higher energies. ${ }^{16}$

The number of charge carriers is commonly conserved across a phase transition and its density is connected to the integrated optical conductivity $\sigma_{x x}=\sigma_{1 x x}+i \sigma_{2 x x}$. In turn, the diagonal element of the dielectric function $\varepsilon_{x x}$ is directly related to $\sigma_{x x}(\omega) \propto i \omega \varepsilon_{x x}(\omega)$. Hence, a good quantity to study the temperature dependence of the charge density is the normalized integrated spectral weight difference,

$$
\Delta W(T)=\frac{\int_{\omega_{1}}^{\omega_{2}}\left[\sigma_{1 x x}(\omega, T)-\sigma_{1 x x}\left(\omega, T_{\text {ref }}\right)\right] d \omega}{\int_{\omega_{1}}^{\omega_{2}} \sigma_{1 x x}\left(\omega, T_{\text {ref }}\right) d \omega},
$$

with $T_{\text {ref }}=450 \mathrm{~K}$. This quantity should vanish in the case of a conservation of the carrier density inside the observed spectral range. Figure 1(d) shows the development of $\Delta W(T)$ for the $\mathrm{AB}$ plane, which is integrated over the spectral range from $\omega_{1}=0.5 \mathrm{eV}$ to $\omega_{2}=4.5 \mathrm{eV}$. The spectral weight is conserved in the investigated spectral range for temperatures between 450 and $110 \mathrm{~K}$. However, below $110 \mathrm{~K}$, an increase in $\Delta W(T)$ is visible. According to Eq. (1), this implies a shift of spectral weight into the observed spectral range for temperatures below $110 \mathrm{~K}$. This spectral weight presumably comes from higher energies as the low energy response remains marginal. ${ }^{16}$ Below $40 \mathrm{~K}$, the spectral weight is constant as can be seen more clearly in the inset of Fig. 1(d).

In order to connect the results of the spectral weight trans-
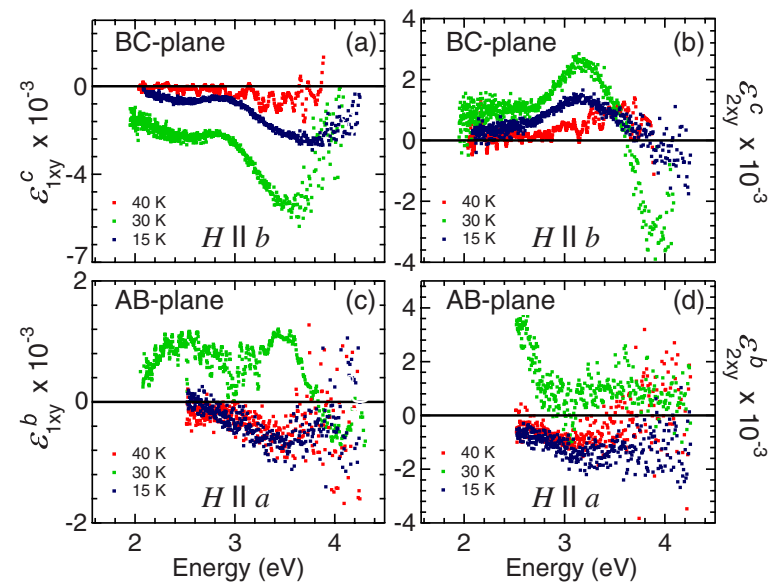

FIG. 2. (Color online) The real $\left(\varepsilon_{1 x y}\right)$ and imaginary $\left(\varepsilon_{2 x y}\right)$ part of the off-diagonal elements of the dielectric tensor for 15 (blue), 30 (green), and 40 (red) of $\mathrm{TbMnO}_{3}$.

fers, as shown in Fig. 1(d), with the development of the magnetic order, we have correlated these data with the temperature dependence of the off-diagonal components $\varepsilon_{x y}$ of the dielectric tensor, which gives information about the spin polarization of the electronic states.

Figure 2 presents $\varepsilon_{x y}$ for three temperatures: 40, 30, and $15 \mathrm{~K}$, which are close to $T_{N}, T_{F}$, and below $T_{F}$, respectively. Figure 2 shows both the real part $\varepsilon_{1 x y}$ (left panel) and the imaginary part $\varepsilon_{2 x y}$ (right panel). The largest temperaturedependent changes were observed for measurements on the BC plane along the $c$ axis, with $H \| b$ [Figs. 2(a) and 2(b)]. In this geometry, a peak in $\varepsilon_{2 x y}$ is observed around $3.2 \mathrm{eV}$; this peak is strongest at $30 \mathrm{~K}$, but is also present at $15 \mathrm{~K}$. Measurements on the $\mathrm{AB}$ plane were also performed for measurements along the $b$ axis with $H \| a$, but the intensity is much weaker [Figs. 2(c) and 2(d)].

For a better understanding of our data, we present a sketch of the spin orientations, with and without an applied magnetic field $H$ [Fig. 3]. For measurements on the BC plane, the sinusoidal (spiral) $J_{S S}$ and antiferromagnetic $J_{A F}$ spin coupling are both present. Along the $a$ axis, the ferromagnetic spin coupling $J_{F M}$ exists. By applying an external magnetic field $H \| b$, we expect a net magnetic moment along

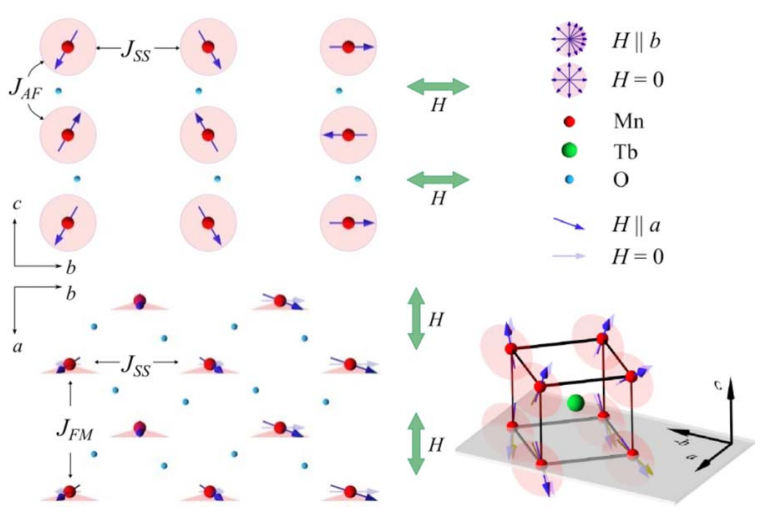

FIG. 3. (Color online) Sketch of the spin orientations for the examined planes. $J_{S S}$ describes the sinusoidal and spiral, $J_{A F}$ the antiferromagnetic, and $J_{F M}$ the ferromagnetic spin couplings. 


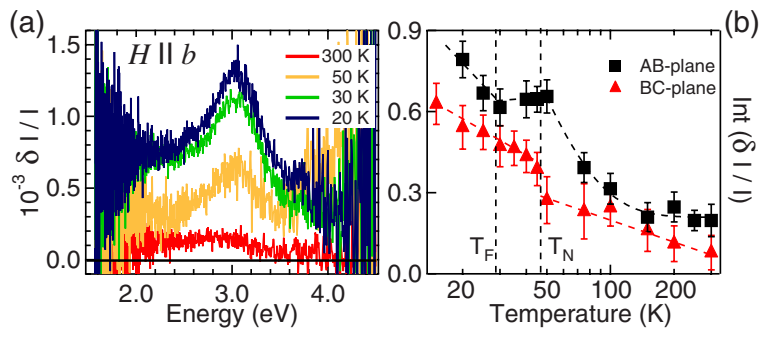

FIG. 4. (Color online) (a) Temperature dependence of the fractional intensity change $\delta I / I$ measured for $H=440 \mathrm{mT}$, which is polarizer position $-30^{\circ}$ and analyzer position $10^{\circ}$. (b) Integrated $\delta I / I$ spectra between 1.75 and $4.0 \mathrm{eV}$ as a function of temperature.

the $b$ axis, as illustrated in the top right picture in Fig. 3. The off-diagonal components of the dielectric tensor $\varepsilon$ are calculated from many $\delta I / I$ measurements for different polarizer and analyzer orientations. ${ }^{8}$ Thus, $\delta I / I$ shows a similar temperature dependence for both planes [Fig. 4].

Figure 4(a) shows the fractional intensity changes $\delta I / I$ as a function of energy, for different temperatures, taken on the BC plane along the $c$ axis and for $H \| b$. For all measurements, the same polarizer orientation $\theta_{1}=-30^{\circ}$ and analyzer orientation $\theta_{2}=+10^{\circ}$ were kept.

These spectra display a peak centered around $3.0 \mathrm{eV}$, which present for all measurements even above $6 T_{N}$. In Fig. 4 (b), the corresponding integrals of each $\delta I / I$ spectrum $(\omega$ $\in[1.75,4.0] \mathrm{eV})$ are shown as a function of temperature for both planes. For measurements on the BC plane (red triangles), the integrated weight between 1.75 and $4.0 \mathrm{eV}$ linearly increases with decreasing temperature but exhibits an abrupt increase at $T_{N}=46 \mathrm{~K}$. For measurements on the AB plane (black squares), a stronger, nonlinear increase in the integrated weight is observed down to $T_{N}$, followed by a constant integrated weight between $T_{N}$ and $T_{F}$. At temperatures below $T_{F}$, at which a spiral-spin-modulation is present in $\mathrm{TbMnO}_{3},{ }^{6}$ our measurements on the AB-plane indicate a systematic increase in the integrated weight. This shows that the off-diagonal components of the dielectric function with $H \| b$ are quite sensitive to the transition into the ferroelectric state, which is in contrast to the diagonal components, which show no sensitivity to the ferroelectric transition [Fig. 1(d)].

With the help of Fig. 3 and the interpretation of the $\delta I / I$ spectra (Fig. 4), we explain the differences between the offdiagonal components for the $\mathrm{AB}$ and $\mathrm{BC}$ planes [Fig. 2]. For both planes, due to the applied magnetic field $H$, a shift of the spin density in the spiral and sinusoidal phase is present. For the $\mathrm{AB}$ plane, a tilt of the spins out of their rotating direction by an external field $H$ exists, but due to the different couplings $\left(J_{F M}, J_{S S}\right.$, and $\left.J_{A F}\right)$ along the corresponding axis, the signals are of different strength. The second phase transition at $T_{F}=29 \mathrm{~K}$ was only observable for the $\mathrm{AB}$ plane [Fig. 4(b)]. In principle, the sinusoidal modulation misses a component parallel to the $a$ axis, which explaines why this phase transition is not apparent in the $\mathrm{BC}$ projection. Due to the pure magnetic coupling, the sinusoidal spiral transition is not observable in standard ellipsometry (see Fig. 1). We would like to emphasize that our interpretation does not rely on the Dzyaloshinski-Moriya interaction, which may induce a persistent ferromagnetic moment along the $c$ axis. ${ }^{17,18}$

By combining standard spectral and magneto-optical ellipsometry, we have studied the charge and spin polarizations of multiferroic $\mathrm{TbMnO}_{3}$. The magneto-optical measurements show changes in the electronic structure for temperatures below $T_{N}$ and $T_{F}$. Furthermore, our data exhibit a sensitivity to the sinusoidal spiral phase transition. The observation of a signal up to $300 \mathrm{~K}$ (approximately $6 T_{N}$ ) is consistent with the formation of short range magnetic ordering well above $T_{N}$.

We thank O. Albrecht for SQUID and J. Strempfer for support at the Laue measurements. We acknowledge financial support by the DFG via Grant No. Ru 773/3-1, GK 1286 and SFB 508, as well as the Helmholtz association contract number VH-FZ-007. We also acknowledge financial support Division of Materials Sciences Under Award number DEFG02-07ER46453.
${ }^{1}$ M. Fiebieg, J. Phys. D 38, R123 (2005).

${ }^{2}$ T. Kimura, T. Goto, H. Shintani, K. Ishizaka, T. Arima, and Y. Tokura, Nature (London) 55, 426 (2003).

${ }^{3}$ M. Kenzelmann, A. B. Harris, S. Jonas, C. Broholm, J. Schefer, S. B. Kim, C. L. Zhang, S.-W. Cheong, O. P. Vajk, and J. W. Lynn, Phys. Rev. Lett. 95, 087206 (2005).

${ }^{4}$ M. Mostovoy, Phys. Rev. Lett. 96, 067601 (2006).

${ }^{5}$ R. Rauer, M. Rübhausen, and K. M. Dörr, Phys. Rev. B 73, 092402 (2006).

${ }^{6}$ N. Aliouane, D. N. Argyriou, J. Strempfer, I. Zegkinoglou, S. Landsgesell, and M. V. Zimmermann, Phys. Rev. B 73, 020102(R) (2006).

${ }^{7}$ H. S. Bennet and E. A. Stern, Phys. Rev. 137, A448 (1965).

${ }^{8}$ R. Rauer, G. Neuber, J. Kunze, J. Bäckström, and M. Rübhausen, Rev. Sci. Instrum. 76, 023910 (2005).

${ }^{9}$ A. Berger and M. R. Pufall, Appl. Phys. Lett. 71, 965 (1997).

${ }^{10}$ V. Perebeinos and P. B. Allen, Phys. Rev. Lett. 85, 5178 (2000).

${ }^{11}$ K. Tobe, T. Kimura Y. Okimoto, and Y. Tokura, Phys. Rev. B 64,
184421 (2001).

${ }^{12}$ R. Krüger, B. Schulz, S. Naler, R. Rauer, D. Budelmann, J. Bäckström, K. H. Kim, S.-W. Cheong, V. Perebeinos, and M. Rübhausen, Phys. Rev. Lett. 92, 097203 (2004).

${ }^{13}$ N. N. Kovaleva, A. V. Boris, C. Bernhard, A. Kulakov, A. Pimenov, A. M. Balbashov, G. Khaliullin, and B. Keimer, Phys. Rev. Lett. 93, 147204 (2004).

${ }^{14}$ M. W. Kim, P. Murugavel, Sachin Parashar, J. S. Lee, and T. W. Noh, New J. Phys. 6, 156 (2004).

${ }^{15}$ M. W. Kim, S. J. Moon, J. H. Jung, Jaejun Yu, Sachin Parashar, P. Murugavel, J. H. Lee, and T. W. Noh, Phys. Rev. Lett. 96, 247205 (2006).

${ }^{16}$ A. Rusydi, R. Rauer, G. Neuber, M. Bastjan, I. Mahns, S. Müller, P. Saichu, B. Schulz, S. G. Singer, A. I. Lichtenstein, G. Stryganyuk, K. Dörr, G. A. Sawatzky, S. L. Cooper, and M. Rübhausen, Phys. Rev. Lett. (to be published).

${ }^{17}$ I. Dzyaloshinskii, J. Phys. Chem. Solids 4, 241 (1958).

${ }^{18}$ T. Moriya, Phys. Rev. 120, 9 (1960). 